\title{
Erratum: High figure of merit magneto-optics from interfacial skyrmions on topological insulators [Phys. Rev. B 98, 024424 (2018)]
}

Tonmoy K. Bhowmick, Amrit De, and Roger K. Lake

Q (Received 21 April 2020; published 8 May 2020)

DOI: 10.1103/PhysRevB.101.209902

The purpose of this erratum is to correct a typographical error in Eq. (8) in our recently published paper. The corrected transfer matrix equation should be

$$
\boldsymbol{S}^{ \pm}=\prod_{j=0}^{N} \frac{1}{t_{j, j+1}^{ \pm}}\left[\begin{array}{cc}
1 & r_{j, j+1}^{ \pm} \\
r_{j, j+1}^{ \pm} & 1
\end{array}\right]\left[\begin{array}{cc}
e^{i \beta_{j+1}^{ \pm}} & 0 \\
0 & e^{-i \beta_{j+1}^{ \pm}}
\end{array}\right] .
$$

Note that does not affect any of the results in the paper, as they were calculated using the correct transfer matrices. 